\title{
Genetic Reconstruction of One Hereditary Nonpolyposis Colorectal Cancer History with Multiple Initiations of the Disease
}

\author{
Olga A. Vostrukhina ${ }^{1,2, *}$, Tatyana A. Shtam ${ }^{1}$, Alexey V. Gulyaev ${ }^{3}$, Kazimir M. Pozharisski ${ }^{4}$ and \\ Vladislav A. Lanzov ${ }^{1,2}$
}

\begin{abstract}
${ }^{I}$ Division of Molecular and Radiation Biophysics, B.P. Konstantinov Petersburg Nuclear Physics Institute (PNPI), Russian Academy of Sciences, Gatchina/St. Petersburg, 188300, Russia; ${ }^{2}$ Research-Education Center Biophysics, St. Petersburg State Polytechnic University and PNPI, St. Petersburg 194021, Russia; ${ }^{3}$ Division of Gastrointestinal Tumors, Prof. N.N. Petrov Research Institute of Oncology, Ministry of Health, St. Petersburg, 197758, Russia and ${ }^{4}$ Laboratory of Immunohistochemistry, Central Research Institute of Roentgenoradiology, Ministry of Health, St. Petersburg, 197758, Russia
\end{abstract}

\begin{abstract}
The scheme of accumulation of genetic alterations in a long-term case of hereditary nonpolyposis colorectal cancer (HNPCC) was reconstructed by the retrospective analysis of postoperative tissue materials. This unique case resulted from a primary lesion in $M L H 1$ gene and revealed two genetic pathways of carcinogenesis in tumors separated by lifetime and localization.
\end{abstract}

\section{INTRODUCTION}

Hereditary nonpolyposis colorectal cancer (HNPCC), also known as the Lynch syndrome, is the most common form of hereditary colorectal cancer [1]. A hallmark of tumors in HNPCC is microsatellite instability (MSI) [2-6] or, in other words, contraction or elongation of microsatellites due to the misalignment of their repetitive nucleotides during DNA replication. Such abnormalities are corrected by human mismatch repair (MMR) system [7]. Since an interconnection between HNPCC, MSI and MMR have been found, it became clear that the MMR deficiency appears to be a specific molecular mechanism responsible for HNPCC.

A germ-line mutation is a primary lesion in HNPCC. Two genes of the MMR system, MLH1 or MSH2, account for about $90 \%$ of all such mutations [1]. Gene $M L H 1$ is an important constituent of MMR system [8,9]. Its product forms two heterodimers with other MutL-like paralogs (MLH1+PMS2 and MLH1+PMS1). All other genes containing repetitive sequences within their coding regions can be considered as equal targets for secondary lesions owing to the mutator phenotype acquired by somatic cells. However, variations in the frequency of these secondary mutations do occur. Among different target genes studied to date, about 20 have been described as those, which accumulate mutations in HNPCC [10-13]. 14 critical genes were used in this study. These include two classes of cancer genes: tumor-suppressors and so called "stability genes". The former class contains genes operating at physiological level including such genes as: IGFIIR $\left(\mathrm{dG}_{8}\right), T G F \beta R I I\left(\mathrm{dA}_{10}\right), B A X\left(\mathrm{dG}_{8}\right)$ [14], E2F4 $\left(\mathrm{dCAG}_{13}\right)$ [15,16], TCF4 $\left(\mathrm{dA}_{9}\right)$, Caspase-5 $\left(\mathrm{dA}_{10}\right)$, MEDI $\left(\mathrm{dA}_{10}\right)$ and RIZ $\left(\mathrm{dA}_{9}\right)$ [10] (numbers in parentheses

*Address correspondence to this author at the Division of Molecular and Radiation Biophysics, B.P. Konstantinov Petersburg Nuclear Physics Institute (PNPI), Russian Academy of Sciences, Gatchina/ St. Petersburg, 188300, Russia; E-mail: oavostr@bpc.spbstu.ru show a microsatellite sequence within the gene). They code, respectively, IGFIIR protein - the insuline-like growth factor II receptor, TGFßRII - transforming growth factor beta type II receptor, BAX - proapoptotic protein (BCL-2 associated $\mathrm{X}$ protein), E2F4 and TCF4 - transcription factors, Caspase5 - apoptosis-related cysteine protease, MED1 - metyl-CpGbinding endonuclease minimizing mutation at methyl-CpG, RIZ - a retinoblastoma protein-interacting zinc finger protein. The class of stability genes is comprised of two genes from MMR system, - MSH6 $\left(\mathrm{dC}_{8}\right)$ and MSH3 $\left(\mathrm{dA}_{8}\right)$ [16], and four genes responsible for recombination repair and

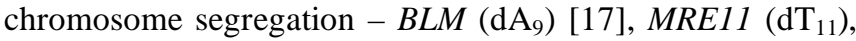
RAD50 $\left(\mathrm{dA}_{9}\right)$ and ATR $\left(\mathrm{dA}_{10}\right)[11,18,19]$, which code, respectively, MSH6 and MSH3 proteins - two of five MutS homologs forming the MSH2+MSH6 and MSH2+MSH3 heterodimers that operate in the MMR system, BLM - a member of the RecQ family of helicases, MRE11 and RAD50 proteins involved in recombination repair of doublestrand DNA breaks, and ATR (ataxia-telangiectasia and RAD3-related) - a member of the phosphatidylinositol kinase-related kinase family.

One of the basic questions of HNPCC tumorigenesis is those initial target gene mutations, which promote the process in each special case. In addition to approaches described earlier [12-15], the comparison of genetic profiles of multiple metachronous cancers growing in the colorectal area of individuals seems to be reasonable. Here, we report a genetic reconstruction of a case of HNPCC with multiple cancers carrying a germinal mutation in $M L H 1$ gene. This approach allows us to reveal the genetic pathways of initiation and progression of an individual case of HNPCC tumorigenesis.

\section{MATERIALS AND METHODS}

\section{Family Pedigree Information and HNPCC History}

This information was obtained from the proband. According to the history, the proband brother and her two sis- 
ters had colorectal cancers and died at 30,52 and 70 years of age, respectively. Her daughter died at age 17 from colorectal carcinoma too. Starting from 38 years of age and during the last 30 years of her life, the proband had eight surgical operations. First operation was on account of adenocarcinoma (AdCa) of the sigmoid colon [tumor 1 (T1)]; three years later - carcinoma of the uterine cervix (T2); eight years after T2 - tubulovillous carcinoma of the transverse colon (T3); eighteen years later - AdCa of the transverse colon (T4); six months later - the same as T4 but with invasion in stomach and duodenum (T5); five months later - the same as T4 but with invasion in stomach and small intestine (T6); and four months later - AdCa of the gall-bladder (T7) with metastases in lymph nodes (T8) and liver (T9). Fig. (1) depicts four tumors from those seven, which were used for genetic analysis. After the fourth tumor resection, the malignancy was diagnosed as the metachronous multiple primary cancer. However, Amsterdam criteria I for HNPCC was met by the proband family, whereas the association of colorectal cancer with extracolonic cancer gave evidence for more detailed diagnosis of Lynch syndrome II [1].
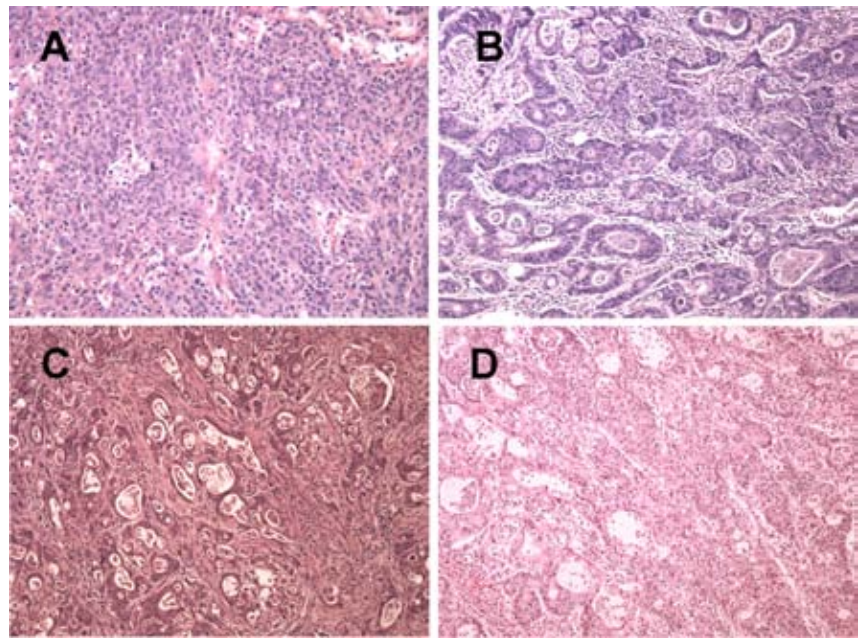

Fig. (1). Photomicrographs of some tumor samples used in the retrospective genetic analysis.

Poorly differentiated AdCa (T1) of the sigmoid colon [A; Hematoxylin and Eosin (H\&E), magnification x100]. Moderately differentiated AdCa (T5) of the transverse colon (B; H\&E, x200). Moderately differentiated $\mathrm{AdCa}$ (T6) forming an extensive conglomerate in the region of ileotransverse anastomosis (C; H\&E, x200). Moderately differentiated AdCa (T7) with the foci of indurations which invade into the muscular stomach wall from serous membrane (D; H\&E, x200).

The tumor and normal tissue paraffin sections were received from Archives of pathologoanatomic laboratory at Prof. N.N. Petrov Research Institute of Oncology in St. Petersburg (Russia).

Unfortunately, the T2 tumor specimen was not available for our testing and the T3 specimen contained a limited number (only 20\%) of cancer cells. Thus, T2 and T3 specimens were not involved in the further retrospective genetic analysis. The paraffin sections of five other proband tumors (T1, T4 - T7) and two metastases of T7 (T8 and T9) were used.

\section{DNA Purification}

Making use of the routine proteinase K-phenol-chloroform extraction method of DNA purification, DNA from the regions of specimen with a large proportion $(60-80 \%)$ of tumor cells was compared with that of normal tissue from the same samples. DNA from tissue specimens from three healthy individuals was used for identification of germinal mutation.

\section{PCR Amplification}

For each pair tumor/norm, specific DNA fragments containing either microsatellites (I) or exons (II) of $M L H 1$, MSH2 and $p 53$ genes were amplified using Programmable Temperature Control System PC-700 ("Astec", Japan). PCR was carried out as follows: 1 cycle of $95^{\circ} \mathrm{C}$ for $4 \mathrm{~min} ; 35$ cycles that included $94^{\circ} \mathrm{C}$ for $30 \mathrm{sec}$ (I) or $1 \mathrm{~min}$ (II), then $60^{\circ}$ for $40 \mathrm{sec}$ (I) or $1 \mathrm{~min}$ (II) or as recommended by [14-16] for each special primer, $72^{\circ}$ for $40 \mathrm{sec}$ (I) or $1 \mathrm{~min}$ (II), and finally one cycle of $72^{\circ}$ for $7 \mathrm{~min}$.

Standard reaction mixture $(20 \mu \mathrm{l})$ contained $250 \mathrm{mM}$ of each dNTP, $10 \mathrm{pM}$ of each primer (from "Syntol", Moscow, Russia), 60-100 ng DNA, 1 unit of Taq DNA polymerase in the original buffer ("Sibenzyme", Novosibirsk, Russia). 56 pairs of primers used in the study have been reported previously [16-18]. Their structures were checked and sometimes corrected in accordance with the NCBI DNA sequence database (GenBank).

\section{Mutation Analysis}

To reveal mutations within the amplified DNA fragments (in pairs: cancer vs. normal cells), two PAGE protocols followed by silver staining were used. Primary search for the mutational changes in DNA was performed by the singlestranded DNA conformation polymorphism (SSCP) [20] that gives the qualitative answer regarding to the presence of mutations. Secondary search for changes in the number of nucleotides was done by the denaturing gel electrophoresis. The latter allows finding of deletions and insertions within microsatellites of the target genes analyzed. When it was necessary, sequence analysis was also done to clarify the results.

\section{RESULTS AND DISCUSSION}

\section{Search for a Germ-Line Mutation}

Both BAT26 $\left(\mathrm{dA}_{26}\right)$ and BAT40 $\left(\mathrm{dA}_{40}\right)$ microsatellite loci [21] showed a high-frequency of MSI in all samples analyzed (Table 1) that was usually expected for tumors originated from defects in the MMR system. In order to find a germ-line mutation 19 exons of $M L H 1$ and 6 exons of MSH2 genes were scanned by PCR-SSCP. When compared with the control specimens from healthy individuals, all tumor and normal tissues of the patient were found to contain damage in the exon 10 of $M L H 1$ gene. Sequencing of corresponding DNA (Fig. 2) revealed a dA/dT deletion in 66 position of the exon 10 of $M L H 1$ (codon 286) that resulted in formation of stop-codon in the third position of exon 11 (codon 295), and, as a consequence, truncation of the MLH1 protein (yielding 294 amino acids instead of 756). As far as we know [22], this germ-line mutation has not been reported previously. 
Table 1. Genetic Profile of Analyzed Tumors

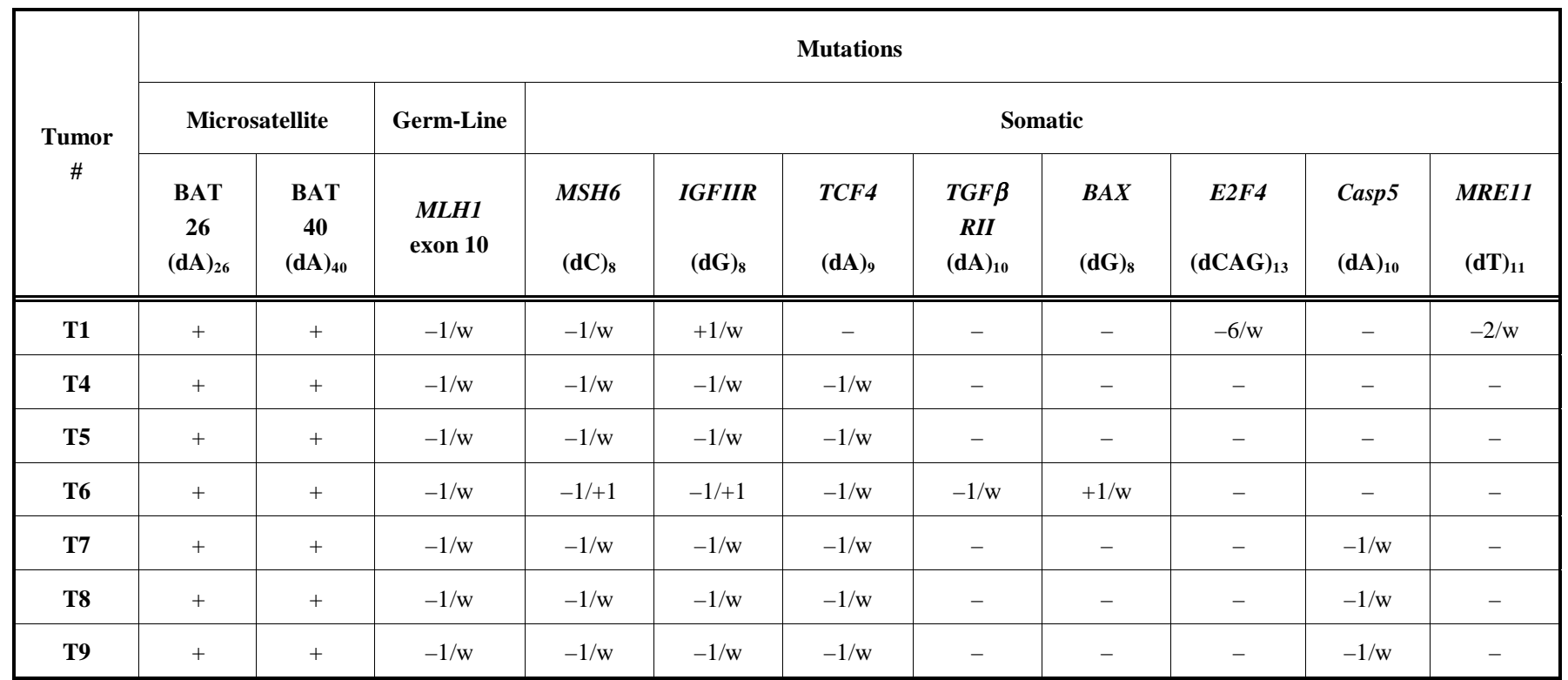

Sign “+” means existence of MSI in BAT26 $\left(\mathrm{dA}_{26}\right)$ or BAT40 $\left(\mathrm{dA}_{40}\right)$ microsatellite loci; sign "_" means absence of any mutations in gene; signs $-1 / \mathrm{w}$ or $+1 / \mathrm{w}$ mean, respectively, the monoallelic deletion or insertion of one nucleotide relative to a wild type (w) allele; sign $-1 /+1$ means biallelic defect of the gene; sign $-6 / \mathrm{w}$ means loss of six nucleotides in one of the alleles of the gene; no one mutation was revealed in the following genes: $M S H 3(\mathrm{dA})_{8}, B L M(\mathrm{dA})_{9}, M E D 1(\mathrm{dA})_{10}, R I Z(\mathrm{dA})_{9}, A T R(\mathrm{dA})_{10}, R A D 50(\mathrm{dA})_{9}$ and $p 53$.

\section{Genetic Profile of Tumors Analyzed}

DNA purified from 14 specimens (cancer and normal tissues of 5 tumors and 2 metastases) was amplified at microsatellite regions located in 14 genes: MSH6, IGFIIR, TCF4, TGFßRII, BAX, E2F4, Caspase-5, MRE11, MSH3, $B L M, M E D 1, R I Z, A T R$ and RAD50. Additionally, five exons (from 5 to 9) of p53 gene [20] with a well-known hot mutagenic activity were amplified too. The amplified DNA fragments from pathological and normal tissues were compared by SSCP for qualitative analysis, and by denaturing gel electrophoresis for quantitative conclusions. Frameshift mutations found in 8 out of 15 tumor suppressor and stability genes (including those shown in the footnote) are presented in Table 1. For the most part the mutations were heterozygous. As expected [1], no mutation was found in the p53 exons that were analyzed. The mutation profile of the subsequently growing tumors was as follows: T1 - MSH6, IGFIIR, E2F4, MRE11, T4 and T5 - MSH6, IGFIIR, TCF4, T6 - MSH6*, IGFIIR* ${ }^{*}, T C F 4, T G F \beta R I I, B A X$; T7 and its metastases T8 and T9 - MSH6, IGFIIR, TCF4 and Caspase-5 ( $\operatorname{sign} *$ shows homozigosity).

Since T6 differed from other tumors by a large number of mutations, five sections of this tumor taken from its center to the edges, or, in other words, from early to late events of tumor progression, were analyzed additionally (Table 2 ). The following genetic profiles were obtained. Section T6(1), that contained the earliest events of tumorigenesis, had mutations in genes MSH6*, IGFIIR, TCF4, TGF $B R I I$; section T6(2) revealed an additional mutation in $B A X$; T6(3) had an additional mutation inactivating both alleles of IGFIIR* $^{*}$ gene. Such a mutation profile was maintained in sections T6(4) and T6(5). Fig. (3) illustrates a typical analysis of tumor /normal (T/N) DNA fragments of different genes amplified from the tissue material of the T6(5) section.

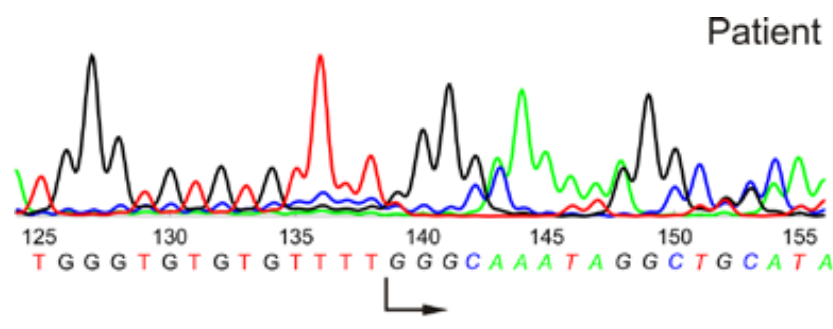

Normal

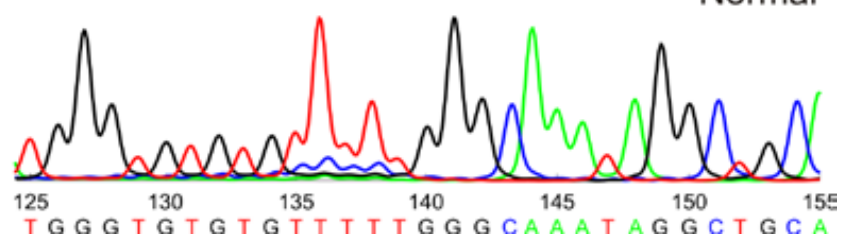

Fig. (2). Nucleotide sequence analysis of $M L H 1$ gene by the PCRsequencing of exon 10 from a patient tissue sample (the top line) and a normal one (the bottom line). The arrow shows a nucleotide shift resulted from the deletion of $\mathrm{T}$ within the $5^{\prime} \rightarrow 3^{\prime}$ strand.

Thus, the section analysis presented appears to be useful for gathering additional information on the genetic basis of HNPCC progression.

\section{Genetic Reconstruction of the HNPCC Initiation and Progression}

The mutation profiles of five subsequent tumors (Table 1) and three subsequent steps of T6 development (Table 2) allow us to reconstruct the genetic basis of tumorigenesis in this HNPCC case. Based on the idea [6] that initial gene alterations would be shared by the most tumors or would be found at early steps of tumor progression, we suggested a possible scheme of consecutive steps in forming the 
frameshift mutations for each tumor mutation profile (Fig. 4).

Table 2. Mutation Profile of 5 Sections* of T6 Tumor

\begin{tabular}{|c|c|c|c|c|c|}
\hline \multirow{2}{*}{$\begin{array}{c}\text { Section } \\
\#\end{array}$} & \multicolumn{5}{|c|}{ Genes } \\
\cline { 2 - 6 } & $\begin{array}{c}\text { MSH6 } \\
(\mathbf{d C})_{\mathbf{8}}\end{array}$ & $\begin{array}{c}\text { TCF4 } \\
(\mathbf{d A})_{\mathbf{9}}\end{array}$ & $\begin{array}{c}\text { IGFIIR } \\
(\mathbf{d G})_{\mathbf{8}}\end{array}$ & $\begin{array}{c}\text { TGFBRII } \\
(\mathbf{d A})_{\mathbf{1 0}}\end{array}$ & $\begin{array}{c}\text { BAX } \\
(\mathbf{d G})_{\mathbf{8}}\end{array}$ \\
\hline \hline T6(1) & $-1 /+1$ & $-1 / \mathrm{w}$ & $-1 / \mathrm{w}$ & $-1 / \mathrm{w}$ & - \\
\hline T6(2) & $-1 /+1$ & $-1 / \mathrm{w}$ & $-1 / \mathrm{w}$ & $-1 / \mathrm{w}$ & $+1 / \mathrm{w}$ \\
\hline T6(3) & $-1 /+1$ & $-1 / \mathrm{w}$ & $-1 /+1$ & $-1 / \mathrm{w}$ & $+1 / \mathrm{w}$ \\
\hline T6(4) & $-1 /+1$ & $-1 / \mathrm{w}$ & $-1 /+1$ & $-1 / \mathrm{w}$ & $+1 / \mathrm{w}$ \\
\hline T6(5) & $-1 /+1$ & $-1 / \mathrm{w}$ & $-1 /+1$ & $-1 / \mathrm{w}$ & $+1 / \mathrm{w}$ \\
\hline
\end{tabular}

*the samples of the T6 tumor cells were taken sequentially from the center of the tissue paraffin section [T6(1)] to its edge [T6(5)]; for other details, see Table 1.

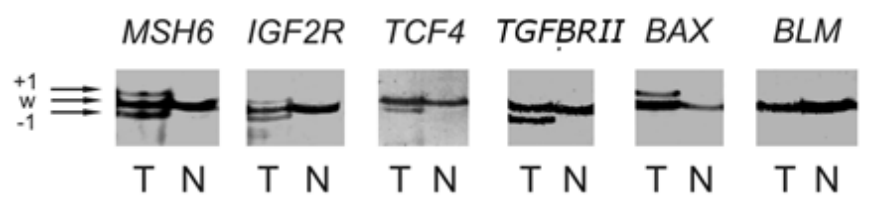

Fig. (3). Denaturing gel electrophoresis of tumor /normal (T/N) amplified DNA fragments from genes MSH6, IGF2R, TCF4, TGFBRII, BAX, and BLM for section T6(5). Sign -1 or +1 indicates, respectively, position of the band with one nucleotide monoallelic deletion or insertion relative to that of a wild type allele (w) found in normal tissue. Analysis of the $B L M$ gene is shown as an example of absence of any nucleotide changes.

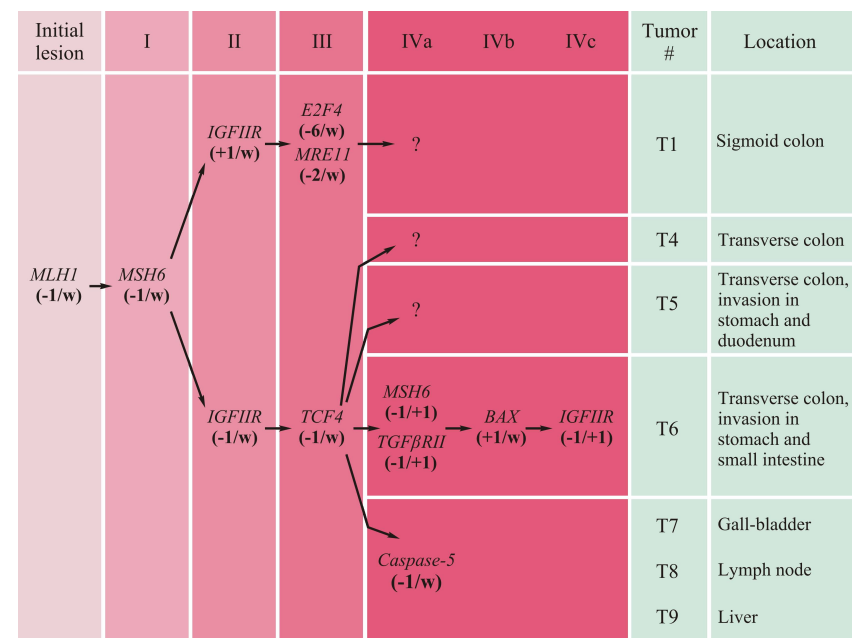

Fig. (4). Accumulation of frameshift mutations in target genes during the initiation and progression of a HNPCC: the case with multiple cancers carrying a germinal mutation in $M L H 1$ gene. The genetic profiles of five AdCas (T1, T4-T7) and two metastases (T8, T9) are presented. I-III, IVa, IVb and IVc - steps of tumorigenesis in individual tumors. For other details, see footnotes to Table $\mathbf{1 .}$

This scheme allows us to speculate that the initiation and progression of all tumors analyzed had a general genetic basis since all tumors demonstrated the similar initial genetic damages: primary germ-line mutation in $M L H 1$, the secondary mutation in MSH6 gene of the MMR system and the inactivation of the insuline-like growth factor II receptor by the frameshift mutation in IGFIIR. However, separated from other tumors by a long time interval (30 years), T1 differs from the subsequent tumors not only in histopathology but in genetics too. The poorly differentiated AdCa (T1) of sigmoid colon is formed by one genetic pathway of the lesion accumulation whereas both moderately differentiated AdCa's of the transverse colon (T4 - T6) and a moderately differentiated AdCa of gall bladder (T7) with its two metastases (T8, T9) belong to another genetic pathway. The latter suggests that tumors T5, T6 and T7 were originated from T4.

The presented data do not pretend for a complete description of all genetic events underlying this special case of HNPCC tumorigenesis. First, we took into consideration only a part of possible mutation events, not analyzing either noncoding DNA or DNA methylation. Second, the mutator phenotype of somatic cells does not exclude an occasional lesion in any site of other oncogenes or tumor-suppressor genes [23]. However, even a limited set of mutation profiles presented here leads to several conclusions: (1) the primary germ-line mutation in $M L H 1$ provokes the secondary mutation in MSH6 gene of the MMR system; (2) the next step of tumorigenesis is inactivation of the insuline-like growth factor II receptor by the frameshift mutation in IGFIIR; (3) an early metastatic cloning (T4 - T7) results in accumulation of different mutations whereas late metastases (T8 and T9) were genetically identical to T7; (4) an additional lesion of the MMR system (the homozygosity of MSH6 defect) results in the burst of mutagenesis observed in T6, that is in good concordance with previous observations [12,24]. A generalization of all these conclusions requires supplementary observations in a number of individual cases.

In sum, molecular genetic analysis of multiple metachronous tumors arising from the presented HNPCC case reveals a definite sequence of genetic lesions that resulted in cancer initiation (steps I - II), additional genetic defects preceding the metastatic process (step III), and the appearance of extra mutations during an individual tumor progression (steps IVa$\mathrm{IVc})$.

\section{CONCLUSION}

Among many MSI-target genes described to date there is a limited number of critical genes, mutations in which predetermine genetic pathways of HNPCC initiation and progression. Here, we proposed an approach to reveal these critical genes. Postoperative tissue materials ( 5 tumors and 2 metastases) from a patient suffering from HNPCC during 30 years met all standards necessary for the retrospective genetic analysis. This case of HNPCC resulted from a primary lesion in $M L H 1$ gene and revealed, at least, two genetic pathways of carcinogenesis in tumors separated by time and localization. Both pathways were initiated by consecutive heterozygous mutations in two genes (MSH6 and IGFIIR). An additional mutation of the mismatch repair system (homozygosity of the MSH6 deficiency) in one of the tumors resulted in the burst of the heterogeneity of the T6 cell progeny.

Thus, the analysis performed made it possible to reconstruct the step-by-step scheme of accumulation of genetic 
alterations in this HNPCC case. One can hope that using the approach for genetic reconstruction of a whole row of individual HNPCC cases can open a new way for generalization of the molecular mechanism of HNPCC initiation and progression.

\section{ACKNOWLEDGMENTS}

We are very grateful to Dr. Vladislav Ten (CRIR) and Georgy Rychkov (PNPI) for help with figure preparations. This work was supported by the Russian Ministry of Education and Science (RNP 2.2.1.1.4663) and St. Petersburg Scientific Center, RAS (2008).

\section{REFERENCES}

[1] Lynch HT, de la Chapell A. Hereditary colorectal cancer. N Engl J Med 2003; 348: 919-32.

[2] Umar A, Boland CR, Terdiman JPS, et al. Revised Bethesda Guidelines for hereditary nonpolyposis colorectal cancer (Lynch syndrome) and microsatellite instability. J Natl Cancer Inst 2004; 96: 261-8

[3] Tsao J-L, Yatabe Y, Salovaara R, et al. Genetic reconstruction of individual colorectal tumor histories. Proc Natl Acad Sci USA 2000; 97: 1236-41.

[4] Kim K-M, Salovaara R, Mecklin J-P, JärvinenHJ, Aaltonen LA, Shibata D. PolyA deletions in hereditary nonpolyposis colorectal cancer. Am J Pathol 2002; 160: 1503-6.

[5] Calabrese P, Mecklin J-P, Järvinen HJ, Aaltonen LA, Tavare S, Shibata D. Number of mutations to different types of colorectal cancer. BMC Cancer 2005; 5: 126.

[6] Hamelin R, Chalastanis A, Colas C, et al. Clinical and molecular consequences of microsatellite instability in human cancers. Bull Cancer 2008; 95: 121-32.

[7] Muller A, Fishel R. Mismatch repair and the hereditary nonpolyposis colorectal cancer syndrome (HNPCC). Cancer Invest 2002; 20: 102-9.

[8] Kim JC, Kim HC, Roh SA. hMLH1 and hMSH2 mutations in families with familial clustering of gastric cancer and hereditary non-polyposis colorectal cancer. Cancer Detect Prev 2001; 25: 50310.

[9] Kolodner RD, Hall NR, Lipford J. Structure of the human MLH1 locus and analysis of a large hereditary nonpolyposis colorectal carcinoma kindred for mlh1 mutations. Cancer Res 1995; 55: 2428.
[10] Duval A, Hamelin R. Mutations at coding repeat sequences in mismatch repair-deficient human cancers: toward a new concept of target genes for instability. Cancer Res 2002; 62: 2447-54.

[11] Kobayashi J, Antoccia A, Tauchi H, Matsuura S, Komatsu K. NBS1 and its functional role in the DNA damage response. DNA Repair (Amst) 2004; 3: 855-61.

[12] Yamaguchi T,IijimaT, Mori T, et al. Accumulation profile of frameshift mutations during development and progression of colorectal cancer from patients with hereditary nonpolyposis colorectal cancer. Dis Colon Rectum 2006; 49: 399-406.

[13] Chung YJ, Park SW, Song JM, et al. Evidence of genetic progression in human gastric carcinomas with microsatellite instability. Oncogene 1997; 15: 1719-26.

[14] Yagi OK, Akiyama Y, Nomizu T, Iwama T, Endo M, Yuasa Y. Proapoptotic gene BAX is frequently mutated in hereditary nonpolyposis colorectal cancers but not in adenomas. Gastroenterology 1998; 114: 268-74.

[15] Biswas S, Chytil A, Washington K, et al. Transforming growth factor beta receptor type II inactivation promotes the establishment and progression of colon cancer. Cancer Res 2004; 64: 4687-92.

[16] Kim JJ, Baek MJ, Kim L, et al. Accumulated frameshift mutations at coding nucleotide repeats during the progression of gastric carcinoma with microsatellite instability. Lab Invest 1999; 79: 1113-20.

[17] Calin G, Ranzani GN, Amadori D, et al. Somatic frameshift mutations in the Bloom syndrome BLM gene are frequent in sporadic gastric carcinomas with microsatellite mutator phenotype. BMC Genet 2001; 2: 14.

[18] Duval A, Reperant M, Compoint A, et al. Target gene mutation profile differs between gastrointestinal and endometrial tumors with mismatch repair deficiency. Cancer Res 2002; 62: 1609-12.

[19] Giannini G, Rinaldi C, Ristori E, et al. Mutations of an intronic repeat induce impaired MRE11 expression in primary human cancer with microsatellite instability. Oncogene 2004; 23: 2640-27.

[20] Steingrimsdottir H, Penhallow J, Farzaneh F, Johnson N, Tavassoli $\mathrm{M}$. Detection of p53 mutations in oral cancer samples using a sensitive PCR-based method. Biochem Soc Trans 1997; 25: 315-8.

[21] Samowitz WS, Slattery ML, Potter JD, Leppert MF. BAT-26 and BAT-40 instability in colorectal adenomas and carcinomas and germline polymorphisms. Am J Pathol 1999; 154: 1637-41.

[22] Stenson PD, Ball EV, Mort M, et al. Human Gene Mutation Database (HGMD). Hum Mutat 2003; 21: 577-81.

[23] Vogelstein B, Kinzler KW. Cancer genes and the pathways they control. Nat Med 2004; 10: 789-99.

[24] Baranovskaya S, Soto JL, Perucho M, Malkosyan SR. Functional significance of concomitant inactivation of hMLH1 and hMSH6 in tumor cells of the microsatellite mutator phenotype. Proc Natl Acad Sci USA 2001; 98: 15107-12. 